\title{
Design and rationale of a 16-week adjunctive randomized placebo-controlled trial of mitochondrial agents for the treatment of bipolar depression
}

\author{
Olivia M. Dean, ${ }^{1,2,3}$ Alyna Turner, ${ }^{1,4}$ Gin S. Malhi, ${ }^{5,6}$ Chee ${ }^{2},{ }^{7}$ Sue M. Cotton, ${ }^{8,9}$ Seetal Dodd, ${ }^{1,3}$ \\ Jerome Sarris, ${ }^{3,10}$ Yuval Samuni, ${ }^{1}$ Michelle Tanious, ${ }^{5,6}$ Nathan Dowling, ${ }^{7}$ Astrid Waterdrinker, ${ }^{1}$ \\ Deidre Smith, ${ }^{7}$ Michael Berk ${ }^{1,2,3,8}$
}

\begin{abstract}
${ }^{1}$ IMPACT Strategic Research Centre, School of Medicine, Deakin University, Geelong, Australia. ${ }^{2}$ Florey Institute for Neuroscience and Mental Health, University of Melbourne, Melbourne, Australia. ${ }^{3}$ University of Melbourne, Melbourne, Australia. ${ }^{4}$ University of Newcastle, Newcastle, Australia. ${ }^{5}$ Discipline of Psychiatry, Sydney Medical School, University of Sydney, Sydney, Australia. ${ }^{6}$ Department of Psychiatry, CADE Clinic, Sydney, Australia. ${ }^{7}$ Department of Psychiatry University of Melbourne, Melbourne, Australia. ${ }^{8}$ Orygen Youth Health Research Centre, Melbourne, Australia. ${ }^{9}$ Centre of Youth Mental Health, University of Melbourne, Melbourne, Australia. ${ }^{10}$ Centre for Human Psychopharmacology, Swinburne University of Technology, Melbourne, Australia.
\end{abstract}

\begin{abstract}
Objective: Bipolar disorder places a significant burden on individuals, caregivers and family, and the broader community. Current treatments are believed to be more effective against manic symptoms, leaving a shortfall in recovery during the depressive phase of the illness. The current study draws on recent evidence suggesting that, in addition to increased oxidative load, alterations in mitochondrial function occur in bipolar disorder.

Methods: This 16-week study aims to explore the potential benefits of N-acetylcysteine (NAC) alone or in combination (CT) with selected nutraceuticals believed to enhance mitochondrial function. The study includes adults diagnosed with bipolar disorder currently experiencing an episode of depression. Participants are asked to take NAC, CT, or placebo in addition to any usual treatments. A postdiscontinuation visit is conducted 4 weeks following the treatment phase.

Results: The primary outcome of the study will be mean change on the Montgomery-Asberg Depression Rating Scale. Secondary outcomes include functioning, substance use, mania ratings, and quality of life. Blood samples will be collected at baseline and week 16 to explore biochemical alterations following treatment.

Conclusion: This study may provide a novel adjunctive treatment for bipolar depression. Analysis of biological samples may assist in understanding the therapeutic benefits and the underlying etiology of bipolar depression.

Trial registration: Australian and New Zealand Clinical Trial Registry ACTRN12612000830897.
\end{abstract}

Keywords: Mitochondria; bipolar disorder; depression; acetylcysteine; oxidative stress

\section{Introduction}

Given the failure of almost all modern trials of antidepressants to demonstrate adequate efficacy in bipolar disorder (BD) and the limited efficacy of mood stabilizers (anticonvulsants and atypical antipsychotics) in the depressive phase of the disorder, the treatment of bipolar depression is widely acknowledged as the major unmet need in the disorder. Yet, individuals with BD spend three times longer in the depressive phase than the manic phase. ${ }^{1}$ Together with depressive mixed states, it is the most lethal phase of the disorder, as it is associated with substantial suicide risk. ${ }^{2}$ Bipolar depression is highly

Correspondence: Michael Berk, IMPACT SRC, School of Medicine, Deakin University, P.O. Box 281, 3220, Geelong, VIC, Australia.

E-mail: mikebe@barwonhealth.org.au

Submitted Dec 16 2013, accepted May 052014. disabling, and causes marked occupational and social impairment. The three largest and most methodologically rigorous modern trials of antidepressants in BD (paroxetine, bupropion, and agomelatine) have all been negative. ${ }^{3-5}$

The etiology of BD remains to be fully elucidated. Therefore, current research is increasingly focusing on novel pathways. For example, new treatments are being sought that focus specifically on pathophysiological aberrations seen in $\mathrm{BD}$. To this end, there is a growing body of evidence that points to a critical role for oxidative stress and mitochondrial dysfunction in BD. The aim of this study is to develop a novel intervention for the depressive phase of $\mathrm{BD}$ which specifically targets these pathways. In particular, two parallel strategies will be studied: a definitive exploration of the use of $\mathrm{N}$-acetylcysteine (NAC), an agent that has robust antioxidant effects, enhances neurogenesis, reduces 
inflammation, and reverses mitochondrial toxicity; and a combination of agents that synergistically target mitochondrial dysfunction. ${ }^{6,7}$

Mitochondria are responsible for energy generation, and are particularly active in oxygen-rich and highly energy dependent tissues, such as the brain. Impaired energy metabolism triggers pro-apoptotic signaling, oxidative damage, and excitotoxicity, and impedes mitochondrial DNA repair. These processes can interact and potentiate one another, which, in turn, results in a continuation of energy depletion. Due to high levels of oxygen metabolism in brain tissue, mitochondria are themselves highly susceptible to oxidative stress. ${ }^{8}$ This then leads to a vicious cycle whereby mitochondrial dysfunction leads to further oxidative stress, in turn causing further damage to the mitochondrion. As such, interventions that improve mitochondrial function by sustaining adenosine triphosphate (ATP) levels could improve neuronal dysfunction and reduce loss. Importantly, interventions that buffer intracellular energy levels and offer neuroprotection may significantly impede the progression of neurological damage, which is an important demonstrated feature of $\mathrm{BD} .^{9}$

There is accumulating evidence demonstrating alterations in mitochondrial homeostasis and, subsequently, function in BD. For example, there is a far higher prevalence of both $\mathrm{BD}$ and depression in people with mitochondrial disease than in the general population. ${ }^{10}$ Changes in brain energy levels and markers of energy metabolism are altered in depression and BD, also implicating a role of mitochondrial changes in these illnesses. For example, resting energy expenditure and maximal aerobic capacity $\left(\mathrm{VO}_{2} \max \right)$ of individuals during the manic phase have been shown to be increased when compared with controls and euthymic participants. ${ }^{11}$ There is also an established body of data from SPECT studies showing increased blood flow in mania and diminished uptake in depression. ${ }^{12}$ Increased phosphomonoester and decreased ATP and phosphocreatine ( $\mathrm{PCr}$, a high energy phosphate) levels have been documented in unmedicated patients with depression. ATP and PCr appear to normalize following acetyl-Lcarnitine (ALC) treatment, and this correlates with improvement on Hamilton Depression Rating Scale (HAM-D) symptom scores. ${ }^{13}$ Furthermore, Kato et al. ${ }^{14}$ reported that frontal lobe $\mathrm{PCr}$ metabolite levels are decreased in patients with severe depression. Subsequent studies suggested that such findings were also seen in patients with bipolar depression in the left frontal region, especially in bipolar II disorder, ${ }^{15}$ and correlated with HAM-D ratings of depression. ${ }^{16}$ Furthermore, photic stimulation-induced reduction of $\mathrm{PCr}$ in the occipital cortex was exaggerated in patients with lithiumresistant $\mathrm{BD} .{ }^{17}$ In addition, there is mounting evidence showing genetic links between BD and mitochondrial dysfunction. ${ }^{18-20}$

The current study has two broad aims. First, we wish to establish further evidence of the efficacy of NAC in the management of $\mathrm{BD}^{21,22}$ As we have previously reported a significant reduction in depressive symptoms associated with NAC in people with $\mathrm{BD},{ }^{21-23}$ a second positive placebo-controlled trial would provide sufficient evidence to consider NAC as a first-line therapy. Second, we aim to develop a combination treatment consisting of agents with known efficacy in mitochondrial disorders. We are using a "systems biology theoretical approach," which suggests that greater efficacy might be attainable using simultaneous and synergistic combinations of agents. These agents will be used adjunctively to augment existing treatments, potentially resulting in better outcomes for people with $\mathrm{BD}$, specifically those experiencing the depressive phase of the illness.

We aim to test this using a 16-week treatment trial that builds on the evidence of mitochondrial dysfunction in BD and will explore the development of a rational, pathophysiologically based intervention for this disorder. This novel project aims to target underlying mitochondrial dysfunction in BD with a purpose-designed, multi-component nutrient formula.

A number of pharmacological treatments and nutritional supplements have previously been used to treat conventional mitochondrial disorders. These include antioxidants (ubiquinone, idebenone, vitamin $\mathrm{C}$, vitamin $\mathrm{E}$, and menadione), agents that specifically improve lactic acidosis (dichloroacetate and dimethylglycine), agents that correct secondary biochemical deficiencies (carnitine and creatine), respiratory chain cofactors (nicotinamide, thiamine, riboflavin, pantothenic acid, pyridoxine, and ubiquinone), and hormones (growth hormone and corticosteroids). ${ }^{24}$ While there are few robust findings (reflecting the nascence of the studies), this literature, to some extent, informs potential treatment options.

\section{$\mathrm{N}$-acetylcysteine}

Robust evidence demonstrates the efficacy of the redox modulator and glutathione precursor NAC as a treatment of BD. Our research group conducted the first Stanley Medical Research Institute-sponsored randomized, placebo-controlled trials of NAC in both schizophrenia and $B D$. In the 6-month schizophrenia trial $(n=140)$, adjunctive NAC was associated with a significant advantage over placebo on the Clinical Global Impression (CGI) scale, the Positive and Negative Symptoms Scale total score, and the Barnes Akathisia Scale. ${ }^{25}$ In the 6-month BD trial $(n=75)$, adjunctive NAC in participants with either BD I or II disorder was associated with significantly superior outcomes on the MADRS. Similar findings were evident on measures of functioning and quality of life, with effect sizes consistently in the large range on almost all outcomes measured. ${ }^{23}$ In both trials, adverse effects did not significantly differ between the NAC and placebo groups.

\section{Ubiquinone (coenzyme Q10)}

This compound is an endogenous biological chemical with some evidence for its use in mitochondrial disorders. ${ }^{8}$ Ubiquinone links mitochondrial complex I and III, transferring electrons between the units, ${ }^{26}$ and 
supplementation may improve the efficiency of this process. Unpublished pilot data on the use of a proprietary compound containing ubiquinone demonstrated improvement in mood symptoms in a non-clinical cohort of a placebo-controlled trial. A number of studies examining the effects of ubiquinone in mitochondrial myopathy, encephalopathy, lactic acidosis, and stroke (MELAS) have demonstrated significant reductions in serum lactate and pyruvate and improvement in brain ATP synthesis and symptomologies at a dosage of 150 $\mathrm{mg} /$ day. ${ }^{27-30}$ The lack of research examining the potential benefits of ubiquinone for treatment of BD is surprising. With evidence of benefits in several other related pathologies ${ }^{31-33}$ and its benign tolerability profile, ubiquinone appears to be an ideal candidate for further research in this area.

\section{Alpha-lipoic acid}

Alpha-lipoic acid (ALA) is suggested to have antioxidant properties and play a crucial role in mitochondrial dehydrogenase reactions. Furthermore, ALA has been shown to be beneficial in many oxidative stress models, such as HIV activation, radiation energy, and diabetes (through inhibition of glycation reactions). ${ }^{34}$

\section{Acetyl-L-carnitine}

ALC is another compound with potential utility in mitochondrial disorders. Carnitine is responsible for transport of fatty acids across the mitochondrial membrane, and plays a role in energy generation via acetyl-coenzyme A. Excitotoxicity and disrupted energy metabolism share free radical-induced oxidative damage as a cause; ALC has shown potential to correct this. ${ }^{35}$ The usefulness of ALC in mood disorders has been suggested by a treatment trial of 204 patients with dysthymia, where ALC was equivalent to amisulpride. ${ }^{36}$ ALC as an adjunct to psychosocial intervention was reported to be useful in the treatment of attention deficit/hyperactivity disorder in 63 boys with fragile $X$ syndrome. ${ }^{37}$ Increased phosphomonoester and decreased ATP and PCr levels have been implicated in depression. These levels were increased and normalized following ALC treatment, and this increase correlated with trends for improvements in symptoms based on HAM-D scores in two unmedicated patients. ${ }^{13}$ The prevalence of metabolic syndrome in BD has been described as "alarmingly high,"38 and increased levels of both lactate and glucose are observed in the cerebrospinal fluid of patients with BD. ${ }^{39}$ L-Carnitine has shown some efficacy in glucose disposal, storage, and uptake in metabolically impaired and in normal healthy adults. ${ }^{40}$

\section{Retinyl palmitate (vitamin A equivalent)}

Retinal palmitate is a highly bioavailable fat-soluble compound and is a common form of vitamin A used in nutraceutical formulas. Vitamin A plays a major role in physiological processes including vision and reproduction, in addition to its specific role in cell differentiation and growth, and, importantly, immune function. Retinol assists in the modulation of the PKC signaling pathway in mitochondria. While its antioxidant profile is well established, there is also evidence that retinol may be an electron carrier. ${ }^{41}$

\section{Vitamin D (cholecalciferol, D3)}

Vitamin $D$ is an antioxidant that may be useful in enhancing mitochondrial function in $\mathrm{BD}$. It plays a predominant role in the regulation of calcium and phosphate metabolism, ${ }^{42}$ which may be particularly important, given the alterations seen in BD. Furthermore, vitamin $\mathrm{D}$ is believed to play a role in the production of melatonin and may be implicated in seasonal affective disorder. ${ }^{43}$ Deficiency of vitamin $D$ has been associated with low mood in a cross-sectional study of older adults. ${ }^{44}$ Vitamin D deficiency is prevalent in psychiatric disorders, and has been linked to the symptoms of depression and schizophrenia. ${ }^{45}$ Furthermore, it has been reported that the risk of developing psychotic symptoms is lower in women in the highest quartile of vitamin $D$ levels, compared with those in the lowest. ${ }^{46}$

\section{Ascorbic acid (vitamin C)}

Ascorbic acid (in the form of calcium ascorbate dihydrate as used in the current study) has possible efficacy in mitochondrial myopathy, ${ }^{47}$ and has a theoretical mechanism of action as an antidepressant in BD through the suppression of vanadium, which is elevated during manic episodes. ${ }^{48,49}$ Ascorbic acid (vitamin C) is also a mediator in L-carnitine production. ${ }^{50}$ Dual administration may strengthen the availability of L-carnitine in addition to its intrinsic properties.

\section{Tocopherol (vitamin E)}

The main function of tocopherol (vitamin E) is to scavenge free radicals and reduce lipid peroxidation, which improves mitochondrial membrane integrity. Studies have suggested that tocopherol and ubiquinone are more efficient when used together. ${ }^{51}$ As such, ascorbic acid and tocopherol could be useful cofactors in a mitochondrial formulation.

\section{B-group vitamins}

Other cofactors associated with improved mitochondrial function include B vitamins, which often work in synergy, and thereby are often best administered as a complex. The water-soluble $B$ vitamins play essential roles in mitochondrial energy production and cellular function by a variety of means. Vitamin B1 (thiamine) functions as a coenzyme in the oxidative decarboxylation of both pyruvate and alpha-ketoglutarate. The use of thiamine in the treatment of some forms of pyruvate dehydrogenase deficiency has been well established. ${ }^{52}$ This use is postulated to improve aerobic glycolysis by enhancing pyruvate decarboxylation. Vitamin B2 (riboflavin) is 
required synergistically with other $B$ vitamins for mitochondrial respiration. ${ }^{53}$ Vitamin B3 (niacin) acts as a precursor for both nicotinamide adenine dinucleotide (NAD/NADH) and nicotinamide adenine dinucleotide phosphate. ${ }^{54}$ Pantothenic acid (vitamin B5) is required for the synthesis of coenzyme A, which, among other roles, is important in the tricarboxylic acid (TCA) cycle. In a study of immortalized T-cell lines, pantothenic acid was shown to prevent loss of mitochondrial membrane potential and also to reestablish ATP synthesis. Levels of catalase, glutathione peroxidase and glutathione reductase were also restored following pantothenic acid treatment. $^{53}$

Vitamin B6 (pyridoxine) is also involved in mitochondrial energy production, and has important effects on antioxidant status, being involved with aminotransferase activity. ${ }^{53,55}$ The major role of $\mathrm{NADH}$ is to transfer electrons from metabolite intermediates to the respiratory chain. Complex I accepts electrons from NADH and passes them to ubiquinone. Nicotinamide increases the cellular NADH and NAD concentration and thereby enhances substrate availability for complex I.

Vitamin B7 (biotin) is part of the B-group complex and is responsible for a variety of biological activities, including promotion of immune and metabolic function, as well as cellular growth. It is a coenzyme for five mitochondrial carboxylases and is essential for growth, development, and normal mitochondrial and cellular functions, including fatty acid oxidation and gluconeogenesis. ${ }^{53}$ Reductions in biotin result in the loss of mitochondrial complex IV, which leads to increased production of oxidative species by the mitochondria.

Vitamin B9 (folic acid) is commonly deficient in hospitalized psychiatric patients. ${ }^{56}$ Deficiency in folic acid impairs synthesis of tetrahydrobiopterin (BH4), which is essential for the synthesis of serotonin and other monoamines involved in the pathogenesis of affective disorders. ${ }^{57}$ Together with vitamin B12 (in the form of cyanocobalamin in the current study), folate plays an essential role in mitochondrial energy production through one-carbon transfer pathways. ${ }^{53}$ B12 is also often deficient in hospitalized psychiatric patients, ${ }^{58}$ and works synergistically with folic acid. ${ }^{59}$ A common finding in vitamin B12 deficiency is pernicious anemia, which may cause a derivative secondary mania. ${ }^{60-62}$ Even in the absence of clinical features of pernicious anemia, neurological signs are often present and, in such cases, may be resolved through B12 supplementation. ${ }^{62-64}$

Although there are many mitochondrial-enhancing agents to consider as potential treatments for BD, for reasons of practicality a number of key agents can be chosen. Additionally, many mitochondrial agents have overlapping mechanisms of action as antioxidants. NAC has been selected as an antioxidant in this trial as it has the best-established efficacy in mental health, and ALC and ubiquinone, for their critical roles in mitochondrial bioenergetics. The cofactor nutrient agents ascorbic acid, $\alpha$-tocopherol, thiamine, niacin, folic acid, and cyanocobalamin were chosen not only because of their individual benefits, but also due to their synergistic effects when administered conjunctively with other mitochondrial agents. Dhitavat et al. ${ }^{65}$ found a synergistic effect against oxidative stress with combined folic acid, tocopherol, and carnitine administration, which provided superior protection against amyloid-beta induced reactive oxygen species in human neuroblastoma cells. In this combination, folic acid was used to maintain endogenous glutathione, a role provided by NAC in our combination. Importantly, all agents used in the present clinical trial are known to have benign adverse event profiles, with adverse effect rates not differing significantly from placebo in previous studies. Finally, the combination has been formulated as not to interact or deteriorate during storage, allowing maximal effects from all components.

\section{Methods}

\section{Study design}

The study is a 16-week, multi-site, randomized, doubleblind, parallel-group trial of a combination therapy (CT), NAC alone, or placebo in the depressive phase of BD. Participants receive 16 weeks of daily treatment, adjunctive to treatment as usual, with assessment visits at baseline, weeks $4,8,12,16$, and 20 (washout), and a phone interview at week 2 (Table 1). Three study sites, in Geelong, Melbourne, and Sydney, Australia, are recruiting participants. The trial is being conducted in accordance with good clinical practice guidelines, and research and ethics committee approval was sought before the start of the study. All components of the active treatment are well tolerated by humans at the doses proposed for use in this study and are currently available for purchase without prescription in Australia.

\section{Trial allocation, sequence generation, and blinding}

Pack number allocation to treatment arm is randomly assigned using permutated block randomization. The computer-generated randomization plan has been developed by an independent researcher utilizing four-to-ablock design. Disposable blister packs containing the trial medications (Medico Paks) are allocated by trial clinicians sequentially. Packs are identical so as to conceal treatment allocation and blinding. To facilitate the double-blinding process, the trial medications (CT, NAC only, and placebo) are dispensed by an independent pharmacist in identical numbers and capsule forms in sealed containers. The placebo containers are specially treated with subtherapeutic levels of NAC dust to produce its characteristic smell (as manufactured in our previous study). The statistician and trial clinicians are blinded to group allocation. Participants are assigned randomly and consecutively to treatment with $\mathrm{CT}$, NAC, or placebo (1:1:1 design) in a double-blind fashion. All participants will remain on treatment as usual (TAU) for the duration of the trial. 
Table 1 Visit schedule

\begin{tabular}{|c|c|c|c|c|c|c|c|c|}
\hline \multirow{2}{*}{$\begin{array}{l}\text { Visit } \\
\text { Phase } \\
\end{array}$} & \multirow{2}{*}{$\begin{array}{c}\text { Visit 1a } \\
\text { Screening }\end{array}$} & \multirow{2}{*}{$\begin{array}{c}\text { Visit } 1 b^{*} \\
\text { Baseline }\end{array}$} & Phone call & Visit 3 & Visit 4 & Visit 5 & Visit 6 & \multirow{2}{*}{$\begin{array}{c}\text { Visit } 7 \\
\text { Washout }\end{array}$} \\
\hline & & & \multicolumn{5}{|c|}{ Treatment phase } & \\
\hline Week/scale & Week 0 & Week 0 & Week 2 & Week 4 & Week 8 & Week 12 & Week 16 & Week 20 \\
\hline Informed consent & $\mathrm{X}$ & - & - & - & - & - & & - \\
\hline MINI-Plus 5.0 & $x$ & - & - & - & - & - & & - \\
\hline Concomitant medication & $x$ & & & & & & & \\
\hline Entrance criteria & $x$ & - & - & - & - & - & & - \\
\hline SAPAS & & - & & $x$ & & & & \\
\hline Family history & & $\mathrm{x}$ & - & - & - & - & & - \\
\hline Psychiatric history & & $\mathrm{X}$ & - & - & - & - & & - \\
\hline Demographic information & & $x$ & & & & & & \\
\hline Habits & - & $x$ & & $\mathrm{X}$ & $x$ & $\mathrm{x}$ & $\mathrm{x}$ & $\mathrm{X}$ \\
\hline Adverse events & - & - & $\mathrm{X}$ & $x$ & $x$ & $x$ & $\mathrm{X}$ & $x$ \\
\hline Alcohol/substance use & - & $\mathrm{x}$ & & $\mathrm{X}$ & $x$ & $x$ & $\mathrm{x}$ & $x$ \\
\hline MADRS with SIGMA & $\mathrm{X}$ & $\mathrm{X}$ & & $\mathrm{X}$ & $\mathrm{X}$ & $\mathrm{X}$ & $\mathrm{X}$ & $x$ \\
\hline BDRS & - & $x$ & & $x$ & $x$ & $x$ & $x$ & $x$ \\
\hline HAM-A & - & $x$ & & $x$ & $x$ & $\mathrm{X}$ & $x$ & $x$ \\
\hline YMRS & - & $X$ & & $x$ & $x$ & $\mathrm{X}$ & $\mathrm{X}$ & $x$ \\
\hline LIFE-RIFT & - & $x$ & & $\mathrm{x}$ & $x$ & $x$ & $x$ & $x$ \\
\hline SOFAS & - & $\mathrm{X}$ & & $\mathrm{X}$ & $\mathrm{X}$ & $\mathrm{X}$ & $\mathrm{X}$ & $\mathrm{X}$ \\
\hline Q-LES-Q (short form) & - & $x$ & & $\mathrm{x}$ & $x$ & $x$ & $x$ & $x$ \\
\hline CGI-BP severity & - & $x$ & & $x$ & $\mathrm{x}$ & $\mathrm{X}$ & $\mathrm{X}$ & $x$ \\
\hline CGI-BP Improvement & & & & $x$ & $\mathrm{X}$ & $\mathrm{X}$ & $\mathrm{X}$ & $x$ \\
\hline PGI & - & - & & $\mathrm{x}$ & $x$ & $\mathrm{x}$ & $x$ & $\mathrm{x}$ \\
\hline Food Frequency Questionnaire & - & & & $\mathrm{X}$ & & & & \\
\hline Qualitative data collection & $\mathrm{x}$ & $\mathrm{x}$ & $\mathrm{x}$ & $x$ & $\mathrm{x}$ & $\mathrm{x}$ & $\mathrm{x}$ & $\mathrm{x}$ \\
\hline Adherence to trial medication & & & $\mathrm{X}$ & $\mathrm{X}$ & $\mathrm{x}$ & $\mathrm{x}$ & $\mathrm{X}$ & $\mathrm{x}$ \\
\hline Blood sampling (30 ml) & - & $\mathrm{X}$ & - & - & - & & $\mathrm{X}$ & - \\
\hline
\end{tabular}

BDRS = Bipolar Depression Rating Scale; CGI-BP = Clinical Global Impression for Bipolar Disorder; HAM-A = Hamilton Anxiety Rating Scale; LIFE-RIFT = Longitudinal Interval Follow-up Evaluation - Range of Impairment Functioning Tool; MADRS = Montgomery-Asberg Depression Scale; MINI-Plus = Mini-International Neuropsychiatric Interview-Plus; PGI = Patient Global Impression; $Q-L E S-Q=Q$ Quality of Life Enjoyment and Satisfaction Questionnaire; SAPAS = Standard Assessment of Personality - Abbreviated Scale; SIGMA = Structured Interview Guide for the Montgomery-Asberg Depression Rating Scale; SOFAS = Social and Occupational Functioning Assessment Scale; YMRS = Young Mania Rating Scale.

* Screening and baseline visits may be conducted together or as two separate visits, depending on the participant's needs.

\section{Treatment regimen}

The treatment regimen, including the composition of each capsule and the agents included in each group, is shown in Table 2. Each participant is required to take a total of 10 capsules/day (five in the morning and five in the evening). To clarify the inclusion of ubiquinone and ALC twice, and the addition of other agents that are not listed in the rationale above, we are using a commercially available product (kindly supplied by Bioceuticals). The manufacturers have provided a product that best fits our rationale.

As the trial is double-blind, each participant will consume the same amount of capsules at each dose time point (twice daily) in all groups. For example, the NAC-alone group is only required to take four capsules/ day to fulfill the dose of NAC. However, for blinding purposes, we have included six placebo capsules, matched to the CT group, to ensure concealment.

\section{Study group}

Two hundred and twenty-five participants (aged 18 years and over) meeting DSM-IV-TR criteria for BD (I, II, or NOS) on a structured clinical interview (Mini-International Neuropsychiatric Interview-Plus) with a current acute depressive episode (MADRS score $\geqslant 20$ ) will be recruited. Participants are recruited through their case clinicians, advertisement, or via private clinicians (e.g., family physicians or specialists). Potential participants are contacted, briefly screened, and a preliminary interview scheduled. All participants are required to provide written informed consent before enrollment.

\section{Inclusion criteria}

To be included, the participants are required to meet DSM-IV-TR criteria for BD (I, II, or NOS), have a current episode of depressive illness with a MADRS score equal to or greater than 20, have the capacity to consent to the study and comply with study procedures, and be using effective contraception if female, sexually active, and of childbearing age. Participants currently under any form of therapy for their BD (psychotropic medication or psychological therapy) are required to have been on a stable dose of that therapy for at least one month prior to randomization. Participants are also required to nominate a current treating physician, and are not eligible to enter the study until one is identified. If there is a delay of $>7$ days between screening and baseline assessments, or between baseline assessment and medication commencement, the MADRS will be administered again to ensure the participant still meets eligibility criteria $(>20)$. 
Table 2 Treatment group composition and dose

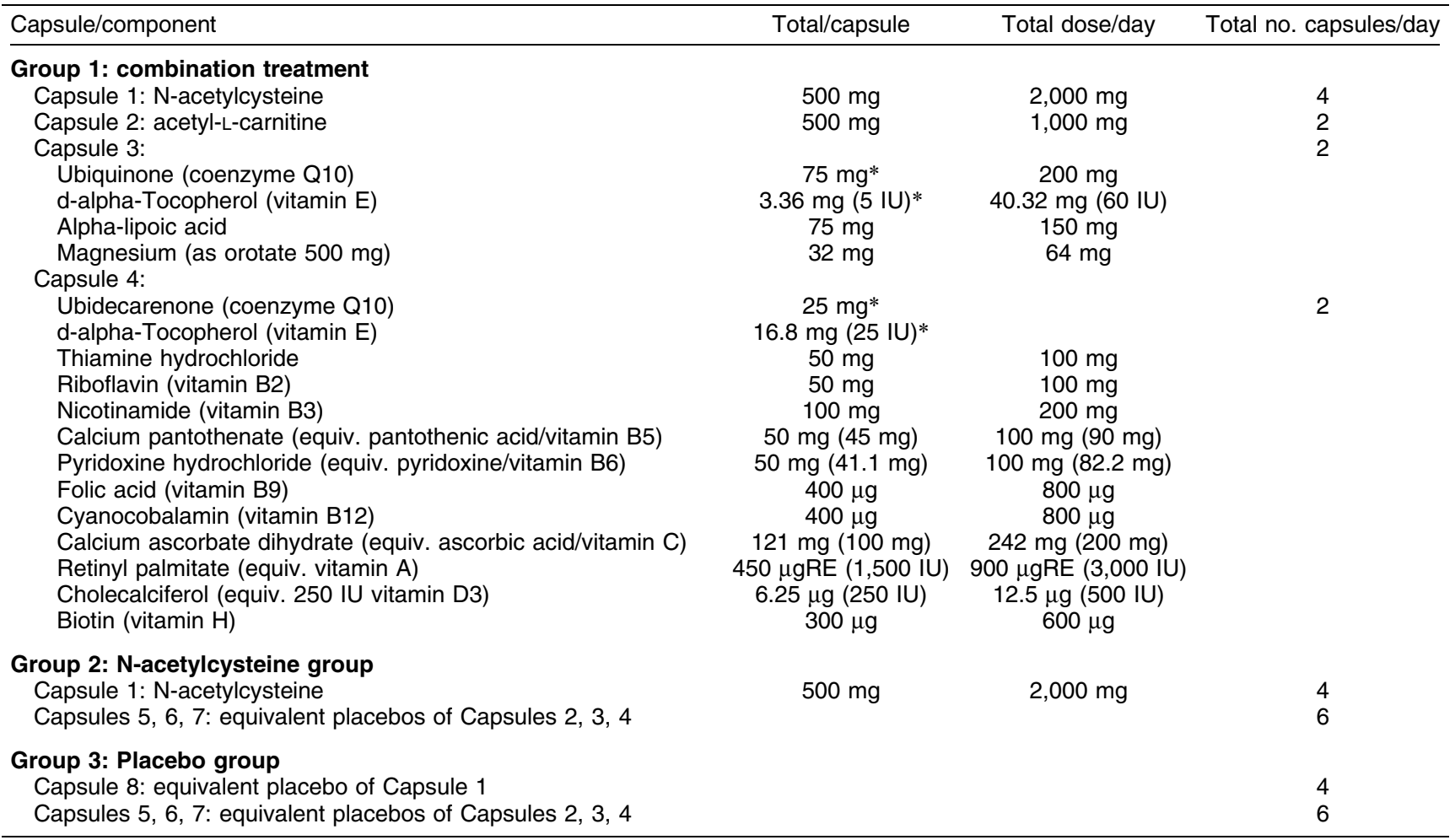

$\mu \mathrm{gRE}=$ micrograms of retinol equivalent.

* Total dose of ubidecarenone is $200 \mathrm{mg}$ and d-alpha-tocopherol is $40.32 \mathrm{mg}$ (60 IU), combining capsules 4 and 5 .

\section{Exclusion criteria}

The following people will be ineligible to take part in the study: individuals with a known or suspected active systemic medical disorder, recent gastrointestinal ulcers or renal stones, individuals who are pregnant or lactating; individuals who have a diagnosis of epilepsy; those who are currently taking $>250 \mathrm{mg}$ of NAC, $>250 \mathrm{mg}$ of ALC, $>25 \mathrm{mg}$ of ubiquinone, or $>200 \mu \mathrm{g}$ of selenium/day (a 1month washout period will be required if participants currently taking these study preparations would like to take part); those currently enrolled in any other intervention study; individuals needing warfarin or phenytoin; individuals who are intolerant to or have had an anaphylactic reaction to any components of the preparation; and those with an inability to comply with either the requirements of informed consent or the treatment protocol. The exclusion of those on selenium is due to its antioxidant properties that are mechanistically similar to those of NAC, which provides cysteine to the glutathione pathway (http://www.genome.jp/kegg-bin/ show_pathway?ec00450+2.9.1.1).

\section{Withdrawal criteria}

Withdrawal from the trial occurs if the participants cease taking their trial medication for 7 consecutive days or if a participant ceases effective contraception or becomes pregnant. Dose changes to existing medications (either increases or decreases in dose) or addition or removal of an agent are acceptable and participants are allowed to continue in the trial. Participants are withdrawn from the study if they withdraw consent or at the discretion of the investigator in case of adverse events or loss to follow-up.

\section{Measurements}

The participants are assessed at baseline using a structured clinical interview, the MINI-Plus. A set of validated outcome measures is completed at baseline and at weeks 4, 8, 12, 16, and 20. The measures include the Montgomery Asberg Depression Rating Scale (MADRS), ${ }^{66}$ HAM-D, ${ }^{67}$ Bipolar Depression Rating Scale (BDRS), ${ }^{68}$ Young Mania Rating Scale (YMRS), ${ }^{69}$ CGl's Improvement and Severity scales, ${ }^{70}$ Patient Global Impression-Improvement subscale, Social and Occupational Functioning Assessment Scale (SOFAS), ${ }^{71}$ Longitudinal Interval Follow-up Evaluation - Range of Impairment Functioning Tool (LIFE RIFT), ${ }^{72}$ and Quality of life Enjoyment and Satisfaction Questionnaire (Q-LesQ) ${ }^{73}$ Information on substance use is also gathered. At week 2 , a phone call is made to assess adherence to trial medication and also to assess any adverse events that may have occurred. In addition, a modified food frequency questionnaire, a physical activity questionnaire, and a Standard Assessment of Personality - Abbreviated Scale $(\text { SAPAS })^{74}$ are administered at week 4. Demographic data are also collected, including, age, weight, height, sex, psychiatric history and duration of illness. 
A purpose-designed qualitative written assessment will be undertaken to explore the effects of treatment on participants' physical and mental health. Clinician case notes from each visit will be used to explore differences over time between the CT, NAC, and placebo groups.

Adherence is monitored using pill counts of returned clinical trial material. Adverse events are recorded, addressed according to medical assessment, and monitored. For consenting participants, blood samples are collected at baseline and at week 16, to be stored and later analyzed for serum levels of pyruvate, lactate, and blood glucose as well as oxidative markers such as cysteine, glutathione, the lipid peroxidation products malondialdehyde and thiobarbituric acid reactive substances, oxidative enzymes (catalase, superoxide dismutase and glutathione peroxidase, GSH-Px), polymorphisms of the GSH-Px, $\gamma$-glutamylcysteine synthetase, and glutamate cysteine ligase subunit genes, and mtDNA polymorphisms. Change in mean MADRS total score is the primary outcome measure.

\section{Data analysis}

Baseline demographic and clinical characteristics will be examined. Measures of central tendency (mean, median) and variability (standard deviation, minimum and maximum) will be estimated for each continuous measure, and proportions estimated for categorical variables. Graphical displays (e.g., histograms and boxplots) will be produced. Transformations will be used when distributional assumptions are not fulfilled for inferential tests. Analysis of the two treatment arms will be compared to placebo and will first be conducted independently of the other. This was not designed as a comparator study to compare the CT and NAC treatments to each other, but rather to explore the benefits of both treatments independently. Comparisons between treatment groups will be made as well. The treatment groups will be compared on baseline demographic and clinical variables using $t$-tests or Mann-Whitney $U$ tests for continuous variables and chi-square tests for categorical variables. If significant baseline group imbalance is detected on a particular variable and that variable is correlated with outcome at $r>0.30$, then it will be included as a covariate in the inferential analyses. Each of the statistical tests above will use a two-tailed alpha level of 0.05 . Mixed-effects linear regression analyses will compare intervention groups on the repeated assessments of the MADRS over 16 weeks. The mixed-effects model will include a random intercept and slope over time and fixed effects for treatment and the stratification variable site. A treatment by time interaction will be used to determine if the change in MADRS score varies between the intervention group over the course of acute treatment. The treatment by time interaction will be evaluated using a likelihood ratio test. Analysis of the qualitative data will use NVivo, with the qualitative differences between $\mathrm{CT}$ and placebo and NAC and placebo being assessed by blinded researchers. We will use an operationalized methodology that we have developed and published. ${ }^{75}$

\section{Training}

All potential raters are required to view the overview training modules and complete reliability and inter-rater reliability assessments. All raters are required to conduct a set of observed interviews on a yearly basis. Any raters failing to meet stringent certification criteria on an ongoing basis will be required to undergo additional remedial training and observation.

\section{Statistical power}

Overall power to detect significant differences between the actual pattern of means will be determined. Assuming a correlation of post-treatment scores with baseline measurements of 0.7 and an effect of the dosage such that the experimental (usual treatment and adjunctive CT) group differs from controls (usual treatment and placebo) by 0.75 standard deviation, power will be maintained above $90 \%$ with 75 subjects in each group. Pairwise comparisons with 75 subjects per group in the three groups would enable effects smaller than 0.6 standard deviations to be detected with a power of $80 \%$. These effect sizes are in the small to moderate range. The experiment would thus be capable of detecting differences between groups of clinical and scientific interest with a total sample size of 225 .

\section{Follow-up of adverse events}

The research clinician will follow up any participant that has completed or withdrawn from the study and that has an ongoing adverse event 30 days after trial medication has ceased. This is based on recommendations from the Orygen Youth Health Research Centre Study Services Unit (internal communication) suggesting that adverse events should be followed up at a time period which is five times the half-life of the agent. Based on available research, cyanocobalamin (vitamin B12) has the longest half-life of any of the agents in the study (6 days). As such, any participants who have an ongoing adverse event at the end of their participation are asked to attend a final phone call or visit 30 days after project completion/ withdrawal.

If a participant withdraws (or is withdrawn) from the study early, they are requested to attend a final faceto-face interview, scheduled to occur as soon as possible, to capture mood symptoms. If the participant is experiencing a current adverse event at that time, they will be asked to be phoned 30 days later to follow up that event. If the participant does not attend a final face-toface visit upon withdrawal, verbal permission will be sought for a final monitoring phone call (30 days postdiscontinuation).

Participants are instructed that, should they have an ongoing adverse event that is concerning them following the completion of the trial, they should contact their 
treating physician rather than wait until the follow-up phone call 30 days later.

\section{Results}

Current data on oxidative stress and mitochondrial dysfunction in $\mathrm{BD}$, together with the results of previous trials, present a compelling indication to examine the role of mitochondrial enhancing agents in BD. To date, we have randomized 81 participants with a view to complete the study in early 2015 .

\section{Discussion}

Bipolar depression remains an unanswered clinical problem with a progressive and relapsing course. Recent advancements in the understanding of neurodegenerative process and disease progression of BD have focused on mitochondrial dysfunction, oxidative stress, neurotrophic factors, and inflammation ${ }^{76}$ rather than on neuroreceptor pathways. Hence, novel treatments are aiming to modulate oxidative, inflammatory, and neurotrophic systems. In particular, attempts to modulate mitochondrial dysfunction seem promising. The current RCT follows a "systems biology theoretical approach" and, to our knowledge, will be the first to evaluate the efficacy of NAC administered alone or with additional nutraceuticals that target mitochondrial dysfunction in participants experiencing current bipolar depression. The advantages of the present study include its double-blind design, the use of face-to-face clinical interviews rather than self-reported questionnaires to generate quantitative and qualitative data, and the inclusion of blood sampling that would allow searching for correlates of biological factors.

The study will also contribute to the understanding of the pathophysiology of bipolar depression. The collection of blood samples at baseline and at the end of the treatment phase will allow investigation of relevant mitochondria-related molecules, as well as those involved in oxidative stress and inflammation. This will not only provide an indication of the mechanisms by which these novel treatments are effective, but might also provide new targets for development of future therapies.

Most importantly, in contrast to many RCTs, the present trial uses commercially-available products which require no additional regulatory approval. Consequently, participants can continue (or commence, if in the placebo group) taking active treatment following the completion of the study. This unique feature is extremely important, as clinicians could use these agents adjunctively to augment existing treatments. Moreover, once recruited into the trial, participants will not be withdrawn even if their medication (i.e., TAU) is altered, reflecting "real-life circumstances."

The consensus in the field of BD research is that the greatest unmet clinical need is bipolar depression. This study provides a novel therapy to target this phase of BD specifically. Given that the agents are safe, affordable, and tolerable, if positive, this study will provide information with a high impact both clinically and scientifically.

\section{Acknowledgements}

The authors would like to acknowledge the support of the National Health and Medical Research Council (NHMRC) and The Cooperative Research Centre for Mental Health. We would also like to acknowledge the service support of Barwon Health, Deakin University, The Melbourne Clinic, University of Melbourne, University of Sydney, and the Royal North Shore Hospital. The trial agents were donated by Bioceuticals and supplied by Catalent and Nutrition Care.

OMD has received grant support from the Brain and Behavior Foundation, Simons Autism Foundation, Stanley Medical Research Institute, and the National Health and Medical Research Council (NHMRC). SD has received research support from the Stanley Medical Research Institute, NHMRC, Beyond Blue, ARHRF, Simons Foundation, and Geelong Medical Research Foundation. JS is sponsored by a CR Roper Fellowship and has received grant support from NHMRC. MB has received grant support from the National Institutes of Health (NIH), Simons Autism Foundation, Cancer Council of Victoria, CRC for Mental Health, Stanley Medical Research Foundation, MBF, NHMRC, Beyond Blue, and Geelong Medical Research Foundation.

\section{Disclosure}

OMD has received grant support from Lilly, and ASBD/ Servier. GSM has received research support from AstraZeneca, Eli Lilly, Organon, Pfizer, Servier and Wyeth; has been a speaker for AstraZeneca, Eli Lilly, Janssen-Cilag, Lundbeck, Pfizer, Ranbaxy, Servier, and Wyeth; and has been a consultant for AstraZeneca, Eli Lilly, Janssen-Cilag, Lundbeck, and, Servier. SD has received research support from Eli Lilly, Glaxo SmithKline, Organon, Mayne Pharma, and Servier; has received speaker's fees and advisory board fees from Eli Lilly; and has received conference travel support from Servier. JS has received either presentation honoraria, travel support, clinical trial grants, or book royalties from Integria Healthcare \& MediHerb, Pfizer, Taki Mai, Pepsico, Bioceuticals \& Blackmores, Soho-Flordis, and Elsevier. MB has received grant support from Bristol-Myers Squibb, Eli Lilly, GlaxoSmithKline, Organon, Novartis, Mayne Pharma, and Servier; has been a speaker for AstraZeneca, Bristol-Myers Squibb, Eli Lilly, GlaxoSmithKline, Janssen-Cilag, Lundbeck, Merck, Pfizer, Sanofi Synthelabo, Servier, Solvay, and Wyeth; has served as a consultant to AstraZeneca, BristolMyers Squibb, Eli Lilly, GlaxoSmithKline, Janssen-Cilag, Lundbeck, and Servier; and is a co-inventor on two provisional patents regarding the use of NAC and related compounds for psychiatric indications, assigned to the Mental Health Research Institute. The other authors report no conflicts of interest. 


\section{References}

1 Judd LL, Akiskal HS, Schettler PJ, Endicott J, Maser J, Solomon DA, et al. The long-term natural history of the weekly symptomatic status of bipolar I disorder. Arch Gen Psychiatry. 2002;59:530-7.

2 Simpson SG, Jamison KR. The risk of suicide in patients with bipolar disorders. J Clin Psychiatry. 1999;60:53-6; discussion 75-6, 113-6.

3 Calabrese JR, Guelfi JD, Perdrizet-Chevallier C, Agomelatine Bipolar Study G. Agomelatine adjunctive therapy for acute bipolar depression: preliminary open data. Bipolar Disord. 2007;9:628-35.

4 McElroy SL, Suppes T, Frye MA, Altshuler LL, Stanford K, Martens $\mathrm{B}$, et al. Open-label aripiprazole in the treatment of acute bipolar depression: a prospective pilot trial. J Affect Disord. 2007;101: 275-81.

5 Sachs GS, Nierenberg AA, Calabrese JR, Marangell LB, Wisniewski SR, Gyulai L, et al. Effectiveness of adjunctive antidepressant treatment for bipolar depression. N Engl J Med. 2007;356:1711-22.

6 Nierenberg AA, Kansky C, Brennan BP, Shelton RC, Perlis R, losifescu DV. Mitochondrial modulators for bipolar disorder: a pathophysiologically informed paradigm for new drug development. Aust N Z J Psychiatry. 2013;47:26-42.

7 Berk M, Malhi GS, Gray LJ, Dean OM. The promise of Nacetylcysteine in neuropsychiatry. Trends Pharmacol Sci. 2013; 34:167-77.

8 Chinnery PF, Majamaa K, Turnbull DM, Thornburn D. Treatment for mitochondrial disorders. Cochrane Database Sys Rev. 2006;25: CD004426.

9 Manji HK, Quiroz JA, Payne JL, Singh J, Lopes BP, Viegas JS, et al. The underlying neurobiology of bipolar disorder. World Psychiatry. 2003;2:136-46.

10 Fattal O, Link J, Quinn K, Cohen BH, Franco K. Psychiatric comorbidity in 36 adults with mitochondrial cytopathies. CNS Spectr. 2007;12:429-38.

11 Caliyurt O, Altiay G. Resting energy expenditure in manic episode. Bipolar Disord. 2009;11:102-6.

12 Baxter LR Jr, Phelps ME, Mazziotta JC, Schwartz JM, Gerner RH, Selin CE, et al. Cerebral metabolic rates for glucose in mood disorders. Studies with positron emission tomography and fluorodeoxyglucose F 18. Arch Gen Psychiatry. 1985;42:441-7.

13 Pettegrew JW, Levine J, Gershon S, Stanley JA, Servan-Schreiber $\mathrm{D}$, Panchalingam $\mathrm{K}$, et al. 31P-MRS study of acetyl-L-carnitine treatment in geriatric depression: preliminary results. Bipolar Disord. 2002;4:61-6.

14 Kato T, Takahashi S, Shioiri T, Inubushi T. Brain phosphorous metabolism in depressive disorders detected by phosphorus-31 magnetic resonance spectroscopy. J Affect Disord. 1992;26:223-30.

15 Kato T, Takahashi S, Shioiri T, Murashita J, Hamakawa H, Inubushi $\mathrm{T}$. Reduction of brain phosphocreatine in bipolar II disorder detected by phosphorus-31 magnetic resonance spectroscopy. J Affect Disord. 1994;31:125-33.

16 Kato T, Shioiri T, Murashita J, Hamakawa H, Takahashi Y, Inubushi $\mathrm{T}$, et al. Lateralized abnormality of high energy phosphate metabolism in the frontal lobes of patients with bipolar disorder detected by phase-encoded 31P-MRS. Psychol Med. 1995;25: 557-66.

17 Murashita J, Kato T, Shioiri T, Inubushi T, Kato N. Altered brain energy metabolism in lithium-resistant bipolar disorder detected by photic stimulated 31P-MR spectroscopy. Psychol Med. 2000;30: 107-15.

18 Kato T, Stine OC, McMahon FJ, Crowe RR. Increased levels of a mitochondrial DNA deletion in the brain of patients with bipolar disorder. Biol Psychiatry. 1997;42:871-5.

19 Sabunciyan S, Kirches E, Krause G, Bogerts B, Mawrin C, Llenos IC et al. Quantification of total mitochondrial DNA and mitochondrial common deletion in the frontal cortex of patients with schizophrenia and bipolar disorder. J Neural Transm. 2007;114:665-74.

20 Fuke S, Kametani M, Kato T. Quantitative analysis of the 4977-bp common deletion of mitochondrial DNA in postmortem frontal cortex from patients with bipolar disorder and schizophrenia. Neurosci Lett. 2008;439:173-7.

21 Berk M, Dean O, Cotton SM, Gama CS, Kapczinski F, Fernandes $\mathrm{BS}$, et al. The efficacy of $\mathrm{N}$-acetylcysteine as an adjunctive treatment in bipolar depression: an open label trial. J Affect Disord. 2011;135:389-94.
22 Berk M, Dean OM, Cotton SM, Gama CS, Kapczinski F, Fernandes $\mathrm{B}$, et al. Maintenance $\mathrm{N}$-acetyl cysteine treatment for bipolar disorder: a double-blind randomized placebo controlled trial. BMC Med. 2012;10:91.

23 Berk M, Copolov DL, Dean O, Lu K, Jeavons S, Schapkaitz I, et al. $\mathrm{N}$-acetyl cysteine for depressive symptoms in bipolar disorder-a double-blind randomized placebo-controlled trial. Biol Psychiatry. 2008;64:468-75.

24 Finsterer J. Treatment of mitochondrial disorders. Eur J Paediatr Neurol. 2010;14:29-44.

25 Berk M, Copolov D, Dean O, Lu K, Jeavons S, Schapkaitz I, et al. Nacetyl cysteine as a glutathione precursor for schizophrenia-a double-blind, randomized, placebo-controlled trial. Biol Psychiatry. 2008;64:361-8.

26 Lenaz G, D'Aurelio M, Merlo Pich M, Genova ML, Ventura B, Bovina $\mathrm{C}$, et al. Mitochondrial bioenergetics in aging. Biochim Biophys Acta. 2000;1459:397-404.

27 Barbiroli B, Frassineti C, Martinelli P, lotti S, Lodi R, Cortelli P, et al Coenzyme Q10 improves mitochondrial respiration in patients with mitochondrial cytopathies. An in vivo study on brain and skeletal muscle by phosphorous magnetic resonance spectroscopy. Cell Mol Biol (Noisy-le-grand). 1997;43:741-9.

28 Brady K, Goldberg JF. Substance abuse and bipolar disorder. In: 9th Annual US Psychiatric and Mental Health Congress; 1996 November 14-17; 1996.

29 Bendahan D, Desnuelle C, Vanuxem D, Confort-Gouny S, FigarellaBranger D, Pellissier JF, et al. 31P NMR spectroscopy and ergometer exercise test as evidence for muscle oxidative performance improvement with coenzyme $Q$ in mitochondrial myopathies. Neurology. 1992;42:1203-8.

30 Tsai G, Goff DC, Chang R, Flood J, Baer L, Coyle JT. Markers of glutamatergic neurotransmission and oxidative stress associated with tardive dyskinesia. Am J Psychiatry. 1998;155:1207-13.

31 Kermode JC, Zheng Q, Cook EP. Fluorescent indicators give biased estimates of intracellular free calcium change in aggregating platelets: Implications for studies with human von willebrand factor. Blood Cells Mol Dis. 1996;22:238-53.

32 Beal MF. Coenzyme Q10 administration and its potential for treatment of neurodegenerative diseases. Biofactors. 1999;9:261-6.

33 Shults CW, Beal MF, Fontaine D, Nakano K, Haas RH. Absorption, tolerability, and effects on mitochondrial activity of oral coenzyme Q10 in parkinsonian patients. Neurology. 1998;50:793-5.

34 Packer L, Tritschler HJ, Wessel K. Neuroprotection by the metabolic antioxidant alpha-lipoic acid. Free Radic Biol Med. 1997;22:359-78.

35 Silva-Adaya D, Perez-De La Cruz V, Herrera-Mundo MN, MendozaMacedo K, Villeda-Hernandez J, Binienda Z, et al. Excitotoxic damage, disrupted energy metabolism, and oxidative stress in the rat brain: antioxidant and neuroprotective effects of $\mathrm{L}$-carnitine. J Neurochem. 2008;105:677-89.

36 Zanardi R, Smeraldi E. A double-blind, randomised, controlled clinical trial of acetyl-L-carnitine vs. amisulpride in the treatment of dysthymia. Eur Neuropsychopharmacol. 2006;16:281-7.

37 Castagnola M, Messana I, Inzitari R, Fanali C, Cabras T, Morelli A, et al. Hypo-phosphorylation of salivary peptidome as a clue to the molecular pathogenesis of autism spectrum disorders. J Proteome Res. 2008;7:5327-32.

38 Marangell LB, Bauer MS, Dennehy EB, Wisniewski SR, Allen MH, Miklowitz DJ, et al. Prospective predictors of suicide and suicide attempts in 1,556 patients with bipolar disorders followed for up to 2 years. Bipolar Disord. 2006;8:566-75.

39 Regenold WT, Phatak P, Marano CM, Sassan A, Conley RR, Kling MA. Elevated cerebrospinal fluid lactate concentrations in patients with bipolar disorder and schizophrenia: implications for the mitochondrial dysfunction hypothesis. Biol Psychiatry. 2009;65: 489-94.

40 Mingrone G, Greco AV, Capristo E, Benedetti G, Giancaterini A, De Gaetano A, et al. L-carnitine improves glucose disposal in type 2 diabetic patients. J Am Coll Nutr. 1999;18:77-82.

41 Hoyos B, Acin-Perez R, Fischman DA, Manfredi G, Hammerling U. Hiding in plain sight: uncovering a new function of vitamin $A$ in redox signaling. Biochim Biophys Acta. 2012;1821:241-7.

42 Ubbenhorst A, Striebich S, Lang F, Lang UE. Exploring the relationship between vitamin $D$ and basic personality traits. Psychopharmacology (Berl). 2011;215:733-7. 
43 Lansdowne AT, Provost SC. Vitamin D3 enhances mood in healthy subjects during winter. Psychopharmacology (Berl). 1998;135: 319-23.

44 Holick MF. Vitamin D deficiency. N Engl J Med. 2007;357:266-81.

45 Eyles DW, Burne TH, McGrath JJ. Vitamin D, effects on brain development, adult brain function and the links between low levels of vitamin D and neuropsychiatric disease. Front Neuroendocrinol. 2013;34:47-64.

46 Hedelin M, Lof M, Olsson M, Lewander T, Nilsson B, Hultman CM, et al. Dietary intake of fish, omega-3, omega- 6 polyunsaturated fatty acids and vitamin $\mathrm{D}$ and the prevalence of psychotic-like symptoms in a cohort of 33,000 women from the general population. BMC Psychiatry. 2010;10:38.

47 Eleff S, Kennaway NG, Buist NR, Darley-Usmar VM, Capaldi RA, Bank WJ, et al. 31P NMR study of improvement in oxidative phosphorylation by vitamins $\mathrm{K} 3$ and $\mathrm{C}$ in a patient with a defect in electron transport at complex III in skeletal muscle. Proc Natl Acad Sci U S A. 1984;81:3529-33.

48 Naylor GJ. Reversal of vanadate-induced inhibition of Na-K ATPase. A possible explanation of the therapeutic effect of carbamazepine in affective illness. J Affect Disord. 1985;8:91-3.

49 Naylor GJ, Smith AH. Vanadium: a possible aetiological factor in manic depressive illness. Psychol Med. 1981;11:249-56.

50 Arrigoni O, De Tullio MC. Ascorbic acid: much more than just an antioxidant. Biochim Biophys Acta. 2002;1569:1-9.

51 Kagan V, Serbinova E, Packer L. Antioxidant effects of ubiquinones in microsomes and mitochondria are mediated by tocopherol recycling. Biochem Biophys Res Commun. 1990;169:851-7.

52 Naito E, Ito M, Takeda E, Yokota I, Yoshijima S, Kuroda Y. Molecular analysis of abnormal pyruvate dehydrogenase in a patient with thiamine-responsive congenital lactic acidemia. Pediatr Res. 1994; 36:340-6.

53 Depeint F, Bruce WR, Shangari N, Mehta R, O'Brien PJ. Mitochondrial function and toxicity: role of the $\mathrm{B}$ vitamin family on mitochondrial energy metabolism. Chem Biol Interact. 2006;163: 94-112.

54 Sauve AA. NAD+ and vitamin B3: from metabolism to therapies. J Pharmacol Exp Ther. 2008;324:883-93.

55 Kannan K, Jain SK. Effect of vitamin B6 on oxygen radicals, mitochondrial membrane potential, and lipid peroxidation in $\mathrm{H} 2 \mathrm{O} 2$ treated U937 monocytes. Free Radic Biol Med. 2004;36:423-8.

56 Hall NC, Carney JM, Plante OJ, Cheng M, Butterfield DA. Effect of 2cyclohexene-1-one-induced glutathione diminution on ischemia/ reperfusion-induced alterations in the physical state of brain synaptosomal membrane proteins and lipids. Neuroscience. 1997;77:283-90.

57 Alpert JE, Fava M. Nutrition and depression: the role of folate. Nutr Rev. 1997;55:145-9.

58 Reynolds EH, Preece JM, Bailey J, Coppen A. Folate deficiency in depressive illness. Br J Psychiatry. 1970;117:287-92.

59 Malouf M, Grimley EJ, Areosa SA. Folic acid with or without vitamin B12 for cognition and dementia. Cochrane Database Syst Rev. 2003;(4):CD004514.

60 Fafouti M, Paparrigopoulos T, Liappas J, Mantouvalos V, Typaldou R, Christodoulou G. Mood disorder with mixed features due to vitamin B(12) and folate deficiency. Gen Hosp Psychiatry. 2002; 24:106-9.

61 Saracaceanu E, Tramoni AV, Henry JM. An association between subcortical dementia and pernicious anemia-a psychiatric mask. Compr Psychiatry. 1997;38:349-51.

62 Storch A, Jost WH, Vieregge P, Spiegel J, Greulich W, Durner J, et al. Randomized, double-blind, placebo-controlled trial on symptomatic effects of coenzyme $Q(10)$ in Parkinson disease. Arch Neurol. 2007;64:938-44.

63 Dommisse J. Subtle vitamin-B12 deficiency and psychiatry: a largely unnoticed but devastating relationship? Med Hypotheses. 1991; 34:131-40.

64 Goggans FC. A case of mania secondary to vitamin B12 deficiency. Am J Psychiatry. 1984;141:300-1.

65 Dhitavat S, Ortiz D, Rogers E, Rivera E, Shea TB. Folate, vitamin E, and acetyl-L-carnitine provide synergistic protection against oxidative stress resulting from exposure of human neuroblastoma cells to amyloid-beta. Brain Res. 2005;1061:114-7.

66 Montgomery SA, Asberg M. A new depression scale designed to be sensitive to change. Br J Psychiatry. 1979;134:382-9.

67 Hamilton M. Development of a rating scale for primary depressive illness. Br J Soc Clin Psychol. 1967;6:278-96.

68 Berk M, Dodd S, Dean OM, Kohlmann K, Berk L, Malhi GS. The validity and internal structure of the Bipolar Depression Rating Scale: data from a clinical trial of $\mathrm{N}$-acetylcysteine as adjunctive therapy in bipolar disorder. Acta Neuropsychiatrica. 2010;22:237-42.

69 Young RC, Biggs JT, Ziegler VE, Meyer DA. A rating scale for mania: reliability, validity and sensitivity. Br J Psychiatry. 1978;133: 429-35.

70 Spearing MK, Post RM, Leverich GS, Brandt D, Nolen W. Modification of the Clinical Global Impressions (CGI) Scale for use in bipolar illness (BP): the CGI-BP. Psychiatry Res. 1997;73:159-71.

71 Morosini PL, Magliano L, Brambilla L, Ugolini S, Pioli R. Development, reliability and acceptability of a new version of the DSM-IV Social and Occupational Functioning Assessment Scale (SOFAS) to assess routine social functioning. Acta Psychiatr Scand. 2000;101:323-9.

72 Keller MB, Lavori PW, Friedman B, Nielsen E, Endicott J, McDonaldScott $P$, et al. The Longitudinal Interval Follow-up Evaluation. A comprehensive method for assessing outcome in prospective longitudinal studies. Arch Gen Psychiatry. 1987;44:540-8.

73 Endicott J, Nee J, Harrison W, Blumenthal R. Quality of Life Enjoyment and Satisfaction Questionnaire: a new measure. Psychopharmacol Bull. 1993;29:321-6.

74 Moran P, Leese M, Lee T, Walters P, Thornicroft G, Mann A. Standardised Assessment of Personality - Abbreviated Scale (SAPAS): preliminary validation of a brief screen for personality disorder. Br J Psychiatry. 2003;183:228-32.

75 Berk M, Munib A, Dean O, Malhi GS, Kohlmann K, Schapkaitz I, et al. Qualitative methods in early-phase drug trials: broadening the scope of data and methods from an RCT of N-acetylcysteine in schizophrenia. J Clin Psychiatry. 2011;72:909-13.

76 Berk M, Kapczinski F, Andreazza AC, Dean OM, Giorlando F, Maes $\mathrm{M}$, et al. Pathways underlying neuroprogression in bipolar disorder: focus on inflammation, oxidative stress and neurotrophic factors. Neurosci Biobehav Rev. 2011;35:804-17. 